\title{
A New Method fOr COMputation of Positive Realizations OF LINEAR DISCRETE-TIME SYSTEMS
}

Submitted: $26^{\text {th }}$ November 2019; accepted: $14^{\text {th }}$ March 2021

\section{Tadeusz Kaczorek}

DOI: 10.14313/JAMRIS/1-2021/1

\begin{abstract}
A new method for computation of positive realizations of given transfer matrices of linear discretetime linear systems is proposed. Sufficient conditions for the existence of positive realizations of transfer matrices are given. A procedure for computation of the positive realizations is proposed and illustrated by an example.
\end{abstract}

Keywords: computation, positive, realization, transfer matrix, linear, discrete-time, system.

\section{Introduction}

A dynamical system is called positive if its trajectory starting from any nonnegative initial state remains forever in the positive orthant for all nonnegative inputs. An overview of state of the art in positive systems theory is given in the monographs $[2,11]$. Variety of models having positive behavior can be found in engineering, economics, social sciences, biology and medicine, etc. $[2,11]$.

The determination of the matrices $A, B, C, D$ of the state equations of linear systems for given their transfer matrices is called the realization problem. The realization problem isa classical problem of analysis of linear systems and has been considered in many books and papers $[9,10,20,22]$. A tutorial on the positive realization problem has been given in the paper [1] and in the books $[2,11]$. The positive minimal realization problem for linear systems without and with delays has been analyzed in $[3,5-7,11-15,18,19,21]$. The existence and determination of the set of Metzler matrices for given stable polynomials have been considered in [8]. The realization problem for positive 2D hybrid systems has been addressed in [17]. For fractional linear systems the realization problem has been considered in [16, 20,22].

In this paper a new method for computation of positive realizations of linear discrete-time systems is proposed. It can be considered as an extension to discrete-time systems of the method presented in [4].

The paper is organized as follows. In section 2 some definitions and theorems concerning the positive discrete-time linear systems are recalled. A new method for computation of positive realizations for single-input single-output linear systems is proposed in section 3 and for multi-input multi-output systems in section 4. Concluding remarks are given in section 5.

The following notation will be used: $\Re$ - the set of real numbers, $\mathfrak{R}^{n \times m}$ - the set of $n \times m$ real matrices, $\mathfrak{R}_{+}^{n \times m}$ - the set of $n \times m$ real matrices with nonnegative entries and $\mathfrak{R}_{+}^{n}=\mathfrak{R}_{+}^{n \times 1}, I_{n}$ - the $n \times n$ identity matrix.

\section{Preliminaries}

Consider the discrete-time linear system

$$
\begin{gathered}
x_{i+1}=A x_{i}+B u_{i}, \\
y_{i}=C x_{i}+D u_{i},
\end{gathered}
$$

where $x_{i} \in \mathfrak{R}^{n}, u_{i} \in \mathfrak{R}^{m}, y_{i} \in \mathfrak{R}^{p}$ are the state, input and output vectors and $A \in \mathfrak{R}^{n \times n}, B \in \mathfrak{R}^{n \times m}, C \in \mathfrak{R}^{p \times n}$, $D \in \mathfrak{R}^{p \times m}$.

Definition 2.1. $[2,11]$ The system (2.1) is called (internally) positive if $x_{i} \in \mathfrak{R}_{+}^{n}$ and $y_{i} \in \mathfrak{R}_{+}^{p}, i \in Z_{+}$for any initial conditions $x_{0} \in \mathfrak{R}_{+}^{n}$ and all inputs $u_{i} \in \mathfrak{R}_{+}^{m}$, $i \in Z_{+}$.

Theorem 2.1. [2, 11] The system (2.1) is positive if and only if

$$
A \in \mathfrak{R}_{+}^{n \times n}, B \in \mathfrak{R}_{+}^{n \times m}, C \in \mathfrak{R}_{+}^{p \times n}, D \in \mathfrak{R}_{+}^{p \times m}
$$

The transfer matrix of the system (2.1) is given by

$$
T(z)=C[I z-A] \quad B+D
$$

Definition 2.2. [1, 22] The matrices (2.2) are called a positive realization of $T(z)$ if they satisfy the equality (2.3).

Definition 2.3. [1, 22] The system (2.1) is called asymptotically stable if the matrix $A$ is a Schur matrix.

Theorem 2.2. [1, 22] The positive realization (2.2) is asymptotically stable if and only if all coefficients of the polynomial

$$
p_{A}(z)=\operatorname{det}\left[I_{n}(z+1)-A\right]=z^{n}+a_{n-1} z^{n-1}+\ldots+a_{1} z+a_{0}
$$

are positive, i.e. $a_{i}>0$ for $i=0,1, \ldots, n-1$.

The positive realization problem can be stated as follows. Given a proper transfer matrix $T(z)$ find its positive realization (2.2).

Theorem 2.3. [22] If (2.2) is a positive realization of (2.3) then the matrices

$$
\bar{A}=P A P^{-1}, \bar{B}=P B, \bar{C}=C P^{-1}, \bar{D}=D
$$


are also a positive realization of (2.3) if and only if the matrix $P \in \mathfrak{R}_{+}^{n \times n}$ is a monomial matrix (in each row and in each column only one entry is positive and the remaining entries are zero).

Proof. Proof follows immediately from the fact that $P^{-1} \in \mathfrak{R}_{+}^{n \times n}$ if and only if $P$ is a monomial matrix.

\section{Positive Realizations of Transfer Functions}

In this section a method for computation of positive realizations $(A, B, C, D)$ of the given transfer function

$$
T(z)=\frac{m_{n} z^{n}+m_{n-1} z^{n-1}+\ldots+m_{1} z+m_{0}}{z^{n}+d_{n-1} z^{n-1}+\ldots+d_{1} z+d_{0}} .
$$

will be proposed.

Using (2.4) we obtain the matrix

$$
D=\lim _{z \rightarrow \infty} T(z)=m_{n}
$$

and the strictly proper transfer function

$$
\bar{T}(z)=T(z)-D=\frac{\bar{m}_{n-1} z^{n-1}+\ldots+\bar{m}_{1} z+\bar{m}_{0}}{z^{n}+d_{n-1} z^{n-1}+\ldots+d_{1} z+d_{0}}
$$

where

$$
\bar{m}_{k}=m_{k}-m_{n} d_{k} \text { for } k=0,1, \ldots, n-1 \text {. }
$$

$$
\begin{aligned}
& C\left[I_{n} z-A\right]^{-1} \\
& =\frac{1}{\left(z-z_{1}\right)\left(z-z_{2}\right) \ldots\left(z-z_{n}\right)}\left[\begin{array}{lllll}
1 & z-z_{1} & \left(z-z_{1}\right)\left(z-z_{2}\right) & \cdots & \left(z-z_{1}\right)\left(z-z_{2}\right) \ldots\left(z-z_{n-1}\right)
\end{array}\right]
\end{aligned}
$$

\section{Lemma 3.1. If}

$$
\begin{gathered}
A=\left[\begin{array}{cccccc}
z_{1} & 0 & 0 & \cdots & 0 & 0 \\
1 & z_{2} & 0 & \cdots & 0 & 0 \\
0 & 1 & z_{3} & & 0 & 0 \\
\vdots & \vdots & \vdots & \ddots & \vdots & \vdots \\
0 & 0 & 0 & \cdots & 1 & z_{n}
\end{array}\right] \in \Re_{+}^{n \times n}, \\
C=\left[\begin{array}{llll}
0 & \cdots & 0 & 1
\end{array}\right] \in \Re_{+}^{1 \times n}
\end{gathered}
$$

has only nonnegative zeros $z_{1}, z_{2}, \ldots, z_{n}$ (not necessardistinct).

In this case it can be written in the form

$$
d(z)=z^{n}-\bar{d}_{n-1} z^{n-1}+\bar{d}_{n-2} z^{n-2}-\bar{d}_{n-3} z^{n-3}+\ldots+(-1)^{n} \bar{d}_{0}
$$

then

Proof. Using (3.6) we obtain

$$
\begin{aligned}
& C\left[I_{n} z-A\right]^{-1}=\left[\begin{array}{llll}
0 & \cdots & 0 & 1
\end{array}\right]\left[\begin{array}{cccccc}
z-z_{1} & 0 & 0 & \cdots & 0 & 0 \\
-1 & z-z_{2} & 0 & \cdots & 0 & 0 \\
0 & -1 & z-z_{3} & \cdots & 0 & 0 \\
\vdots & \vdots & \vdots & \ddots & \vdots & \vdots \\
0 & 0 & 0 & \cdots & -1 & z-z_{n}
\end{array}\right]^{-1} \\
& =\frac{1}{\left(z-z_{1}\right)\left(z-z_{2}\right) \ldots\left(z-z_{n}\right)}\left[\begin{array}{lllll}
1 & z-z_{1} & \left(z-z_{1}\right)\left(z-z_{2}\right) & \cdots & \left.\left(z-z_{1}\right)\left(z-z_{2}\right) \ldots\left(z-z_{n-1}\right)\right]
\end{array}\right.
\end{aligned}
$$

since

$$
C\left[I_{n} z-A\right]_{a d}=\left[\begin{array}{lllll}
1 & z-z_{1} & \left(z-z_{1}\right)\left(z-z_{2}\right) & \cdots & \left(z-z_{1}\right)\left(z-z_{2}\right) \ldots\left(z-z_{n-1}\right)
\end{array}\right]
$$

and (3.7) holds. $\square$

Theorem 3.1. There exists the positive realization

$$
A=\left[\begin{array}{cccccc}
z_{1} & 0 & 0 & \cdots & 0 & 0 \\
1 & z_{2} & 0 & \cdots & 0 & 0 \\
0 & 1 & z_{3} & & 0 & 0 \\
\vdots & \vdots & \vdots & \ddots & \vdots & \vdots \\
0 & 0 & 0 & \cdots & 1 & z_{n}
\end{array}\right] \in \Re_{+}^{n \times n}, \quad B=\left[\begin{array}{c}
b_{1} \\
b_{2} \\
\vdots \\
b_{n}
\end{array}\right] \in \Re_{+}^{n}, \quad C=\left[\begin{array}{llll}
0 & \cdots & 0 & 1
\end{array}\right] \in \Re_{+}^{1 \times n}, \quad D=m_{n}
$$

of the transfer function (3.1) if the denominator (3.4) has nonnegative zeros $z_{1}, z_{2}, \ldots, z_{n}, \bar{m}_{k} \geq 0, k=0,1, \ldots$, $n-1$ (at least one $\bar{m}_{k}>0$ ) and $m_{n} \geq 0$.
Proof. If $z_{k} \geq 0, k=0,1, \ldots, n$ then $A \in \Re_{+}^{n \times n}$. To simplify the notation let us assume that $n=4$. Using Lemma 3.1 for $n=4$ we obtain 


$$
\begin{aligned}
& C\left[I_{4} Z-A\right]^{-1} B=\left[\begin{array}{llll}
0 & 0 & 0 & 1
\end{array}\right]\left[\begin{array}{cccc}
z-z_{1} & 0 & 0 & 0 \\
-1 & z-z_{2} & 0 & 0 \\
0 & -1 & z-z_{3} & 0 \\
0 & 0 & -1 & z-z_{4}
\end{array}\right]_{a d}\left[\begin{array}{l}
b_{1} \\
b_{2} \\
b_{3} \\
b_{4}
\end{array}\right] \\
& =\left[\begin{array}{lllll}
1 & z-z_{1} & \left(z-z_{1}\right)\left(z-z_{2}\right) & \cdots & \left(z-z_{1}\right)\left(z-z_{2}\right)\left(z-z_{3}\right)
\end{array}\right]\left[\begin{array}{l}
b_{1} \\
b_{2} \\
b_{3} \\
b_{4}
\end{array}\right] \\
& =b_{1}-z_{1} b_{2}+z_{1} z_{2} b_{3}-z_{1} z_{2} z_{3} b_{4}+\left[b_{2}-\left(z_{1}+z_{2}\right) b_{3}+\left(z_{1}\left(z_{2}+z_{3}\right)+z_{2} z_{3}\right) b_{4}\right] \\
& +\left[b_{3}-\left(z_{1}+z_{2}+z_{3}\right) b_{4}\right] z^{2}+b_{4} z^{3} \\
& =\bar{m}_{3} z^{3}+\bar{m}_{2} z^{2}+\bar{m}_{1} z+\bar{m}_{0}
\end{aligned}
$$

and

$$
\left[\begin{array}{cccc}
1 & -z_{1} & z_{1} z_{2} & -z_{1} z_{2} z_{3} \\
0 & 1 & -z_{1}-z_{2} & z_{1}\left(z_{2}+z_{3}\right)+z_{2} z_{3} \\
0 & 0 & 1 & -z_{1}-z_{2}-z_{3} \\
0 & 0 & 0 & 1
\end{array}\right]\left[\begin{array}{l}
b_{1} \\
b_{2} \\
b_{3} \\
b_{4}
\end{array}\right]=\left[\begin{array}{c}
\bar{m}_{0} \\
\bar{m}_{1} \\
\bar{m}_{2} \\
\bar{m}_{3}
\end{array}\right] .
$$

Multiplying the second row of (3.11) by $z_{1}$ and adding to its first row, then multiplying third row by $z_{1}+z_{2}$ and adding to its second row we obtain

$$
\begin{gathered}
{\left[\begin{array}{cccc}
1 & 0 & -z_{1}^{2} & -z_{1}^{2}\left(z_{2}+z_{3}\right) \\
0 & 1 & 0 & -z_{1}^{2}-\left(z_{1}+z_{2}\right) z_{3} \\
0 & 0 & 1 & -z_{1}-z_{2}-z_{3} \\
0 & 0 & 0 & 1
\end{array}\right]\left[\begin{array}{l}
b_{1} \\
b_{2} \\
b_{3} \\
b_{4}
\end{array}\right]=} \\
=\left[\begin{array}{c}
\bar{m}_{0}+z_{1} \bar{m}_{1} \\
\bar{m}_{1}+\left(z_{1}+z_{2}\right) \bar{m}_{2} \\
\bar{m}_{2} \\
\bar{m}_{3}
\end{array}\right]
\end{gathered}
$$

From (3.12) it follows that

$$
\begin{gathered}
b_{4}=\bar{m}_{3} \geq 0, \\
b_{3}=\bar{m}_{2}+\left(z_{1}+z_{2}+z_{3}\right) \bar{m}_{3} \geq 0, \\
b_{2}=\bar{m}_{1}+\left(z_{1}+z_{2}\right) \bar{m}_{2}+\left[z_{1}^{2}+\left(z_{1}+z_{2}\right) z_{3}\right] \bar{m}_{3} \geq 0, \\
b_{1}=z_{1}^{2} \bar{m}_{2}+z_{1}^{2}\left(z_{2}+z_{3}\right) \bar{m}_{3} \geq 0
\end{gathered}
$$

and the matrix $B \in \mathfrak{R}_{+}^{4}$. By assumption $D=m_{n} \geq 0$. Therefore, there exists the positive realization (3.9) of the transfer function (3.1) if the denominator (3.4) has nonnegative zeros and $\bar{m}_{k} \geq 0, k=0,1, \ldots, n-1$, $m_{n} \geq 0$. $\square$

Remark 3.1. The positive realization (3.9) is asymptotically stable (Schur) if $z_{k}<1$ for $k=0,1, \ldots, n$.

Example 3.1. Compute the positive realization (3.9) of the transfer function

$$
T(z)=\frac{2 z^{3}-2.6 z^{2}+2.88 z-0.384}{z^{3}-1.8 z^{2}+1.04 z-0.192} .
$$

Using (3.2) and (3.14) we obtain

$$
D=\lim _{z \rightarrow \infty} T(z)=2
$$

and

$$
\begin{aligned}
\bar{T}(z) & =T(z)-D=\frac{z^{2}+0.8 z}{z^{3}-1.8 z^{2}+1.04 z-0.192}= \\
& =\frac{z(z+0.8)}{(z-0.4)(z-0.6)(z-0.8)} \\
& =\frac{\bar{m}_{2} z^{2}+\bar{m}_{1} z}{z^{3}-\bar{d}_{2} z^{2}+\bar{d}_{1} z-\bar{d}_{0}},
\end{aligned}
$$

where $\bar{m}_{2}=1, \bar{m}_{1}=0.8, \bar{m}_{0}=0$ and $z_{1}=0.4, z_{2}=0.6$, $z_{3}=0.8$.

In this case we have

$$
A=\left[\begin{array}{ccc}
z_{1} & 0 & 0 \\
1 & z_{2} & 0 \\
0 & 1 & z_{3}
\end{array}\right]=\left[\begin{array}{ccc}
0.4 & 0 & 0 \\
1 & 0.6 & 0 \\
0 & 1 & 0.8
\end{array}\right], C=\left[\begin{array}{lll}
0 & 0 & 1
\end{array}\right]
$$

and

$$
\begin{aligned}
& {\left[\begin{array}{ccc}
1 & -z_{1} & z_{1} z_{2} \\
0 & 1 & -z_{1}-z_{2} \\
0 & 0 & 1
\end{array}\right]\left[\begin{array}{l}
b_{1} \\
b_{2} \\
b_{3}
\end{array}\right] }=\left[\begin{array}{ccc}
1 & -0.4 & 0.24 \\
0 & 1 & -1 \\
0 & 0 & 1
\end{array}\right]\left[\begin{array}{l}
b_{1} \\
b_{2} \\
b_{3}
\end{array}\right]= \\
&=\left[\begin{array}{l}
\bar{m}_{0} \\
\bar{m}_{1} \\
m_{2}
\end{array}\right]=\left[\begin{array}{c}
0 \\
0.8 \\
1
\end{array}\right] .
\end{aligned}
$$

Solving (3.18) we obtain

$$
B=\left[\begin{array}{l}
b_{1} \\
b_{2} \\
b_{3}
\end{array}\right]=\left[\begin{array}{c}
0.48 \\
1.8 \\
1
\end{array}\right]
$$

The positive asymptotically stable realization of the transfer function (3.14) is given by (3.17), (3.19) and (3.15).

It is easy to check that the matrices

$$
\begin{gathered}
\bar{A}=\left[\begin{array}{ccc}
z_{1} & 1 & 0 \\
0 & z_{2} & 1 \\
0 & 0 & z_{3}
\end{array}\right]=\left[\begin{array}{ccc}
0.4 & 1 & 0 \\
0 & 0.6 & 1 \\
0 & 0 & 0.8
\end{array}\right], \\
\bar{B}=\left[\begin{array}{l}
0 \\
0 \\
1
\end{array}\right], \bar{C}=\left[\begin{array}{lll}
0.48 & 1.8 & 1
\end{array}\right]
\end{gathered}
$$

are also the positive asymptotically stable realization of (3.16).

In general case we have the following theorem.

Theorem 3.2. There exists positive realization

$$
\begin{gathered}
\bar{A}=\left[\begin{array}{cccccc}
z_{1} & 1 & 0 & \cdots & 0 & 0 \\
0 & z_{2} & 1 & \cdots & 0 & 0 \\
\vdots & \vdots & \vdots & \ddots & \vdots & \vdots \\
0 & 0 & 0 & \cdots & z_{n-1} & 1 \\
0 & 0 & 0 & \cdots & 0 & z_{n}
\end{array}\right] \in \Re_{+}^{n \times n}, \\
\bar{B}=\left[\begin{array}{c}
0 \\
0 \\
\vdots \\
1
\end{array}\right] \in \Re_{+}^{n}, \\
\bar{C}=\left[\begin{array}{llll}
c_{1} & c_{2} & \cdots & c_{n}
\end{array}\right] \in \mathfrak{R}_{+}^{1 \times n}, \quad D=m_{n}
\end{gathered}
$$

of the transfer function (3.1) if the denominator (3.4) has nonnegative zeros $z_{1}, z_{2}, \ldots, z_{n}, \bar{m}_{k} \geq 0, k=0,1, \ldots, n-1$ (at least one $\bar{m}_{k}>0$ ) and $m_{n} \geq 0$. 
Proof. The proof is similar (dual) to the proof of Theorem 3.1.

\section{Extension of the Method to MIMO Systems}

In this section the method presented in section 3 will be extended to multi-input multi-output (MIMO) linear systems. To simplify the notation two-input two-output systems will be considered.

The problem can be stated as follows. Given the proper transfer matrix

$$
\begin{gathered}
T(z)=\left[\begin{array}{ll}
T_{11}(z) & T_{12}(z) \\
T_{21}(z) & T_{22}(z)
\end{array}\right], \\
T_{i k}(z)=\frac{m_{i k n} z^{n}+\ldots+m_{i k 1} z+m_{i k 0}}{z^{n}+d_{i k n-1} z^{n-1}+\ldots+d_{i k 1} z+d_{i k 0}}, \\
i, k=1,2
\end{gathered}
$$

find its positive realization $(A, B, C, D)$ such that

$$
T(z)=C\left[I_{n} z-A\right]^{-1} B+D .
$$

Using

$$
D=\lim _{z \rightarrow \infty} T(z)
$$

find the matrix $D \in \mathfrak{R}_{+}^{2 \times 2}$ and the strictly proper transfer matrix

$$
\bar{T}(z)=T(z)-D=C\left[I_{n} z-A\right]^{-1} B=\left[\begin{array}{ll}
\frac{\bar{m}_{11}(z)}{d_{1}(z)} & \frac{\bar{m}_{12}(z)}{d_{1}(z)} \\
\frac{\bar{m}_{21}(z)}{d_{2}(z)} & \frac{\bar{m}_{22}(z)}{d_{2}(z)}
\end{array}\right]
$$

where

$$
d_{i}(z)=z^{n}+d_{i n-1} z^{n-1}+\ldots+d_{i 1} z+d_{i 0}, \quad i=1,2
$$

is the least common denominator of $T_{k}(z), i, k=1,2$ and $z_{i 1}, z_{i 2}, \ldots, z_{i n}, i=1,2$ are nonnegative zeros of $(4.4 \mathrm{~b})$ and

$$
\bar{m}_{i k}(z)=\bar{m}_{i k n-1} z^{n-1}+\ldots+\bar{m}_{i k 1} z+\bar{m}_{i k 1}+\bar{m}_{i k 0}, i=1,2 .
$$

The matrix $A$ of the desired positive realization has the form

$$
A=\left[\begin{array}{cc}
A_{1} & 0 \\
0 & A_{2}
\end{array}\right]=\operatorname{blockdiag}\left[A_{1} \quad A_{2}\right],
$$

where

$$
A=\left[\begin{array}{cccccc} 
& 0 & 0 & & 0 & 0 \\
1 & & 0 & & 0 & 0 \\
\vdots & \vdots & \vdots & \ddots & \vdots & \vdots \\
0 & 0 & 0 & & \text { in } & 0 \\
0 & 0 & 0 & & 1 & \text { in }
\end{array}\right], i=1,2
$$

The matrices $B$ and $C$ have the forms

$$
B=\left[\begin{array}{ll}
B_{11} & B_{12} \\
B_{21} & B_{22}
\end{array}\right], B_{i k}=\left[\begin{array}{c}
b_{i k 1} \\
b_{i k 2} \\
\vdots \\
b_{i k n_{i}}
\end{array}\right] \in \mathfrak{R}_{+}^{n_{i}}, i, k=1,2
$$

$$
\begin{gathered}
C=\text { blockdiag }\left[\begin{array}{ll}
C_{1} & C_{2}
\end{array}\right], \\
C_{i}=\left[\begin{array}{llll}
0 & \cdots & 0 & 1
\end{array}\right] \in \Re_{+}^{1 \times n_{i}}, i=1,2 .
\end{gathered}
$$

The entries of $B_{i k}, i, k=1,2$ can be calculated in the same way as the entries of $B$ in section 3. Therefore, we have the following theorem.

Theorem 4.1. There exists the positive realization given by (4.5), (4.6) and (4.3) of the transfer matrix (4.1) if

1) $D \in \Re_{+}^{2 \times 2}$ (defined by (4.3));

2) The denominators (4.4b), $i=1,2$ have

nonnegative zeros.

Proof. If $z_{i k} \geq 0, \quad i=1,2, \quad k=0,1, \ldots, n$ then $A \in \mathfrak{R}_{+}^{\left(n_{1}+n_{2}\right) \times\left(n_{1}+n_{2}\right)}$. In a similar way as in proof of Theorem 3.1 it can be shown that $B \in \Re_{+}^{\left(n_{1}+n_{2}\right) \times 2}$ if the denominators (4.4b), $i=1,2$ have nonnegative zeros. The matrix $C$ defined by (4.4b) has always nonnegative entries. Therefore, the realization given by (4.5), (4.6) and (4.3) is positive if $D \in \mathfrak{R}_{+}^{2 \times 2}$. .

If the conditions of Theorem 4.1 are satisfied then the following procedure can be used for computation of the positive realization for given transfer matrix (4.1).

\section{Procedure 4.1.}

Step 1. Knowing $T(z)$ and using (4.3) and (4.4a) compute the matrix $D$ and the strictly proper transfer matrix $\bar{T}(z)$.

Step 2. Compute the zeros $z_{i j,}, i=1,2, j=1, \ldots, n$ of the polynomials (4.4b) and find the matrix (4.5).

Step 3. Using the procedure presented in section 3 compute the matrix $B \in \Re_{+}^{\left(n_{1}+n_{2}\right) \times 2}$.

Step 4. Write the desired positive realization given by (4.5), (4.6) and (4.3).

Example 4.1. Compute the positive realization of the transfer matrix

$$
T(z)=\left[\begin{array}{c}
\frac{2 z^{2}-1.2 z+1}{z^{2}-1.1 z+0.3} \\
\frac{3 z+0.8}{z-0.4}
\end{array}\right] .
$$

Using Procedure 4.1 we obtain the following:

Step 1. Using (4.3), (4.2) and (4.8) we obtain

$$
\bar{T}(s)=T(s)-D=\left[\begin{array}{c}
\frac{z+0.4}{z^{2}-1.1 s+0.3} \\
\frac{2}{z-0.4}
\end{array}\right]
$$

and

Step 2. The zeros of the polynomial

$$
d_{1}(z)=z^{2}-1.1 z+0.3
$$

are $z_{11}=0.5, z_{12}=0.6$ and the polynomial

$$
d_{2}(z)=z-0.4
$$

has only one zero $z_{21}=0.4$.

Therefore, the matrix $A$ has the form

$$
A=\left[\begin{array}{cc}
A_{1} & 0 \\
0 & A_{2}
\end{array}\right]=\left[\begin{array}{ccc}
0.5 & 0 & 0 \\
1 & 0.6 & 0 \\
0 & 0 & 0.4
\end{array}\right] .
$$

and 
Step 3. In this case

$$
\begin{gathered}
B=\left[\begin{array}{l}
B_{1} \\
B_{2}
\end{array}\right], B_{1}=\left[\begin{array}{l}
b_{11} \\
b_{12}
\end{array}\right], B_{2}=\left[b_{13}\right] \\
\text { and } \\
B_{1}=\left[\begin{array}{cc}
1 & -z_{11} \\
0 & 1
\end{array}\right]^{-1}\left[\begin{array}{c}
\bar{m}_{10} \\
\bar{m}_{11}
\end{array}\right]=\left[\begin{array}{cc}
1 & 0.5 \\
0 & 1
\end{array}\right]\left[\begin{array}{c}
0.4 \\
1
\end{array}\right]=\left[\begin{array}{c}
0.9 \\
1
\end{array}\right], \\
B_{2}=2 .
\end{gathered}
$$

Thus, we have

$$
B=\left[\begin{array}{c}
0.9 \\
1 \\
2
\end{array}\right] \text { and } C=\left[\begin{array}{cc}
C_{1} & 0 \\
0 & C_{2}
\end{array}\right]=\left[\begin{array}{ccc}
0 & 1 & 0 \\
0 & 0 & 1
\end{array}\right] \text {. }
$$

Step 4. The desired positive realization of (4.8) is given by (4.12), (4.14) and (4.9).

It is easy to verify that the matrices

$$
\begin{gathered}
\bar{A}=\left[\begin{array}{ccc}
0.5 & 0 & 0 \\
1 & 0.6 & 0 \\
0 & 0 & 0.4
\end{array}\right], \bar{B}=\left[\begin{array}{l}
0 \\
1 \\
1
\end{array}\right], \\
\bar{C}=\left[\begin{array}{ccc}
0.9 & 1 & 0 \\
0 & 0 & 2
\end{array}\right], D=\left[\begin{array}{l}
2 \\
3
\end{array}\right]
\end{gathered}
$$

are also the (dual) positive realization of (4.8).

Remark 4.1. To the presented method the dual method based on the least common denominator for each column of $T(z)$ can be also applied.

Remark 4.2. By Theorem 2.3 if the matrices $A, B$, $C, D$ are a positive realization of $T(z)$ then the matrices $P A P^{-1}, P B, C P^{-1}, D$ are also its positive realization for any monomial matrix $P$.

\section{Conclusion}

A new method for computation of positive realizations of transfer matrices of discrete-time linear systems has been proposed. Sufficient conditions for the existence of the positive realizations have been established (Theorems 3.1, 3.2 and 4.1). A procedure for computation of the positive realizations has been proposed and illustrated by a numerical example (Example 4.1). The presented method can be extended to linear fractional systems.

\section{AUTHOR}

Tadeusz Kaczorek - Faculty of Electrical Engineering, Białystok University of Technology, Wiejska 45D, 15-351 Białystok, Poland, e-mail: kaczorek@ee.pw. edu.pl.

\section{REFERENCES}

[1] L. Benvenuti and L. Farina, "A tutorial on the positive realization problem", IEEE Transactions on Automatic Control, vol. 49, no. 5, 2004, 651-664, 10.1109/TAC.2004.826715.

[2] L. Farina and S. Rinaldi, Positive Linear Systems: Theory and Applications, Wiley-Interscience, 2000.

[3] T. Kaczorek, "A modified state variable diagram method for determination of positive realizations of linear continuous-time systems with delays", International Journal of Applied Mathematics and Computer Science, vol. 22, no. 4, 2012, 897-905, 10.2478/v10006-012-0066-x.

[4] T. Kaczorek, "A new method for determination of positive realizations of linear continuous-time systems", Bulletin of the Polish Academy of Sciences: Technical Sciences, vol. 66, no. 5, 2018, 605611, 10.24425/BPAS.2018.124276.

[5] T. Kaczorek, "A realization problem for positive continuous-time systems with reduced numbers of delays", International Journal of Applied Mathematics and Computer Science, vol. 16, no. 3, 2006, 325-331.

[6] T. Kaczorek, "Computation of positive stable realizations for linear continuous-time systems", Bulletin of the Polish Academy of Sciences: Technical Sciences, vol. 59, no. 3, 2011, 273-281, 10.2478/v10175-011-0033-y.

[7] T. Kaczorek, "Computation of realizations of discrete-time cone-systems", Bulletin of the Polish Academy of Sciences: Technical Sciences, vol. 54, no. 3, 2006, 347-350.

[8] T. Kaczorek, "Existence and determination of the set of Metzler matrices for given stable polynomials", International Journal of Applied Mathematics and Computer Science, vol. 22, no. 2, 2012, 389-399, 10.2478/v10006-012-0029-2.

[9] T. Kaczorek, Linear Control Systems: Analysis of Multivariable Systems, Wiley, 1992.

[10] T. Kaczorek, Polynomial and Rational Matrices, Springer London, 2007, 10.1007/978-1-84628605-6.

[11] T. Kaczorek, Positive 1D and 2D Systems, Springer-Verlag, 2002, 10.1007/978-1-4471-0221-2.

[12] T. Kaczorek, "Positive minimal realizations for singular discrete-time systems with delays in state and delays in control", Bulletin of the Polish Academy of Sciences: Technical Sciences, vol. 53, no. 3, 2005, 293-298. 
[13] T. Kaczorek, "Positive stable realizations of fractional continuous-time linear systems". In: Proc. Conf. Int. Inf. and Eng. Syst., 2012.

[14] T. Kaczorek, "Positive stable realizations for fractional descriptor continuous-time linear systems", Archives of Control Sciences, vol. 22, no. 3, 2012, 303-313, 10.2478/v10170-011-0026-y.

[15] T. Kaczorek, "Positive stable realizations with system Metzler matrices", Archives of Control Sciences, vol. 21, no. 2, 2011, 167-188, 10.2478/ v10170-010-0038-z.

[16] T. Kaczorek, "Realization problem for positive fractional continuous-time systems". In: 2008 16th Mediterranean Conference on Control and Automation, 2008, 1008-1015, 10.1109/ MED.2008.4602000.

[17] T. Kaczorek, "Realization problem for positive 2D hybrid systems", COMPEL - The International Journal for Computation and Mathematics in Electrical and Electronic Engineering, vol. 27, no. 3, 2008, 613-623, 10.1108/03321640810861061.

[18] T. Kaczorek, "Realization problem for positive discrete-time systems with delays", Systems Science, vol. 30, no. 4, 2004, 17-30.

[19] T. Kaczorek, "Realization problem for positive multivariable discrete-time linear systems with delays in the state vector and inputs", International Journal of Applied Mathematics and Computer Science, vol. 16, no. 2, 2006, 169-174.

[20] T. Kaczorek, Selected Problems of Fractional Systems Theory, Springer Berlin Heidelberg, 2011, 10.1007/978-3-642-20502-6.

[21] T. Kaczorek and M. Busłowicz, "Minimal realization for positive multivariable linear systems with delay", International Journal of Applied Mathematics and Computer Science, vol. 14, no. 2, 2004, 181-187.

[22] T. Kaczorek and L. Sajewski, The Realization Problem for Positive and Fractional Systems, Springer International Publishing, 2014, 10.1007/978-3319-04834-5. 